\title{
Exercises for adolescent idiopathic scoliosis (Review)
}

Romano M, Minozzi S, Bettany-Saltikov J, Zaina F, Chockalingam N, Kotwicki T, MaierHennes A, Negrini S

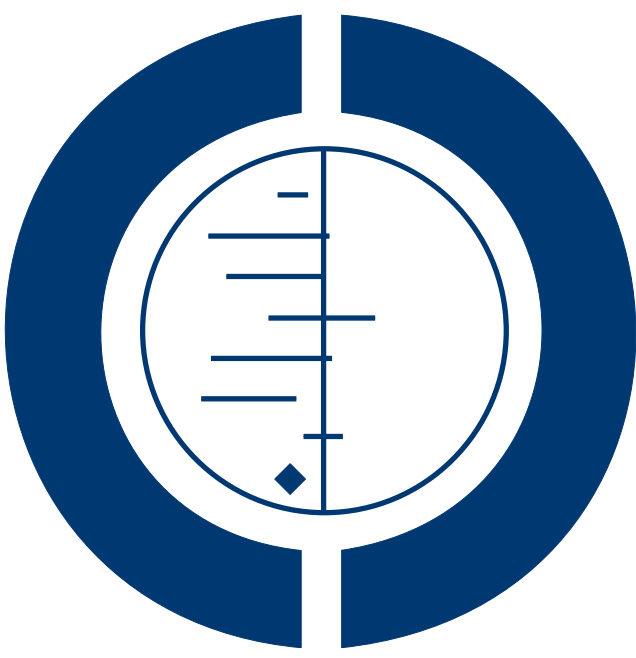

THE COCHRANE COLLABORATION ${ }^{\circledR}$

This is a reprint of a Cochrane review, prepared and maintained by The Cochrane Collaboration and published in The Cochrane Library 2012, Issue 8

http://www.thecochranelibrary.com

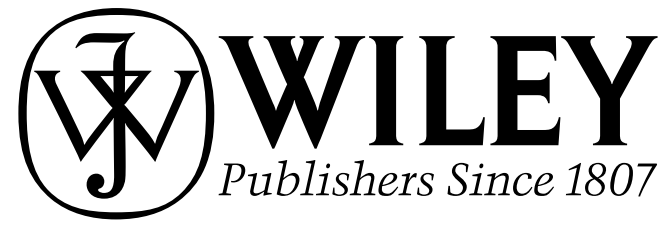

Exercises for adolescent idiopathic scoliosis (Review)

Copyright $\odot 2012$ The Cochrane Collaboration. Published by John Wiley \& Sons, Ltd. 
TABLE OF CONTENTS

HEADER . . . . . . . . . . . . . . . . . . . . . . . . . . . . . . . . . . . . 1

ABSTRACT . . . . . . . . . . . . . . . . . . . . . . . . . . . . . . . . . . . . . . . . . . . . . . .

PLAIN LANGUAGE SUMMARY . . . . . . . . . . . . . . . . . . . . . . . . . . . . . . . . . . . .

SUMMARY OF FINDINGS FOR THE MAIN COMPARISON $\quad$. . . . . . . . . . . . . . . . . . . . . . . . . .

BACKGROUND . . . . . . . . . . . . . . . . . . . . . . . . . . . . . . . . . . . . . 3

OBJECTIVES . . . . . . . . . . . . . . . . . . . . . . . . . . . . . . . . . . . . . . . . . . . .

METHODS . . . . . . . . . . . . . . . . . . . . . . . . . . . . . . . . . . . 5

RESUlTS . . . . . . . . . . . . . . . . . . . . . . . . . . . . . . . . . . . . . 8

Figure 1. . . . . . . . . . . . . . . . . . . . . . . . . . . . . . . . . . . . . . 9

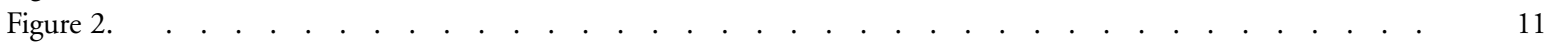

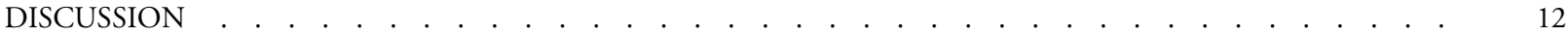

AUTHORS' CONCLUSIONS . . . . . . . . . . . . . . . . . . . . . . . . . . . . . . . . . . . . . .

ACKNOWLEDGEMENTS . . . . . . . . . . . . . . . . . . . . . . . . . . . . . . . . . . . . . . . . 14

REFERENCES . . . . . . . . . . . . . . . . . . . . . . . . . . . . . . . . . . . . . . 14

CHARACTERISTICS OF STUDIES . . . . . . . . . . . . . . . . . . . . . . . . . . . . . . . . 16

DATA AND ANALYSES . . . . . . . . . . . . . . . . . . . . . . . . . . . . . . . . . . . 22

APPENDICES . . . . . . . . . . . . . . . . . . . . . . . . . . . . . . . . . . . . . . 22

HISTORY . . . . . . . . . . . . . . . . . . . . . . . . . . . . . . . . . . . . . . . 30

CONTRIBUTIONS OF AUTHORS . . . . . . . . . . . . . . . . . . . . . . . . . . . . . . . . . . . . . . . .

DECLARATIONS OF INTEREST . . . . . . . . . . . . . . . . . . . . . . . . . . . . . . . . . . . . . . . .

SOURCES OF SUPPORT . . . . . . . . . . . . . . . . . . . . . . . . . . . . . . . . . . . . . . . . . . . .

Exercises for adolescent idiopathic scoliosis (Review)

Copyright $\odot 2012$ The Cochrane Collaboration. Published by John Wiley \& Sons, Ltd. 


\title{
[Intervention Review]
}

\section{Exercises for adolescent idiopathic scoliosis}

Michele Romano ${ }^{1}$, Silvia Minozzi ${ }^{2}$, Josette Bettany-Saltikov ${ }^{3}$, Fabio Zaina ${ }^{1}$, Nachiappan Chockalingam ${ }^{4}$, Tomasz Kotwicki ${ }^{5}$, Axel Maier-Hennes ${ }^{6}$, Stefano Negrini ${ }^{7}$

${ }^{1}$ ISICO (Italian Scientific Spine Institute), Milan, Italy. ${ }^{2}$ Department of Epidemiology, Lazio Regional Health Service, Rome, Italy. ${ }^{3}$ School of Health and Social Care, University of Teeside, Middlesbrough, UK. ${ }^{4}$ Faculty of Health, Staffordshire University, Stoke on Trent, UK. ${ }^{5}$ Department of Pediatric Orthopedics and Traumatology, University of Medical Sciences, Poznan, Poland. ${ }^{6}$ Scoliosis Rehabilitation Centre, Bad Sobernheim, Germany. ${ }^{7}$ Physical and Rehabilitation Medicine, University of Brescia - Don Gnocchi Foundation Milan, Brescia, Italy

Contact address: Michele Romano, ISICO (Italian Scientific Spine Institute), Via Roberto Bellarmino 13/1, Milan, 20141, Italy. michele.romano@isico.it.

Editorial group: Cochrane Back Group.

Publication status and date: New, published in Issue 8, 2012.

Review content assessed as up-to-date: 30 March 2011.

Citation: Romano M, Minozzi S, Bettany-Saltikov J, Zaina F, Chockalingam N, Kotwicki T, Maier-Hennes A, Negrini S. Exercises for adolescent idiopathic scoliosis. Cochrane Database of Systematic Reviews 2012, Issue 8. Art. No.: CD007837. DOI: 10.1002/14651858.CD007837.pub2.

Copyright (C) 2012 The Cochrane Collaboration. Published by John Wiley \& Sons, Ltd.

\begin{abstract}
A B S T R A C T
Background

Adolescent idiopathic scoliosis (AIS) is a three-dimensional deformity of the spine. While AIS can progress during growth and cause a surface deformity, it is usually not symptomatic. However, in adulthood, if the final spinal curvature surpasses a certain critical threshold, the risk of health problems and curve progression is increased. The use of scoliosis-specific exercises (SSE) to reduce progression of AIS and postpone or avoid other more invasive treatments is controversial.
\end{abstract}

\section{Objectives}

To evaluate the efficacy of SSE in adolescent patients with AIS.

\section{Search methods}

The following databases (up to 30 March 2011) were searched with no language limitations: CENTRAL (The Cochrane Library 2011, issue 2), MEDLINE (from January 1966), EMBASE (from January 1980), CINHAL (from January 1982), SportDiscus (from January 1975), PsycInfo (from January 1887), PEDro (from January 1929). We screened reference lists of articles and also conducted an extensive handsearch of grey literature.

\section{Selection criteria}

Randomised controlled trials and prospective cohort studies with a control group comparing exercises with no treatment, other treatment, surgery, and different types of exercises.

\section{Data collection and analysis}

Two review authors independently selected studies, assessed risk of bias and extracted data.

Exercises for adolescent idiopathic scoliosis (Review)

Copyright $\odot 2012$ The Cochrane Collaboration. Published by John Wiley \& Sons, Ltd. 


\section{Main results}

Two studies (154 participants) were included. There is low quality evidence from one randomised controlled study that exercises as an adjunctive to other conservative treatments increase the efficacy of these treatments (thoracic curve reduced: mean difference (MD) 9.00, (95\% confidence interval (CI) 5.47 to 12.53 ); lumbar curve reduced:MD 8.00, (95\% CI 5.08 to 10.92 )). There is very low quality evidence from a prospective controlled cohort study that scoliosis-specific exercises structured within an exercise programme can reduce brace prescription (risk ratio (RR) 0.24, (95\% CI 0.06 to 1.04) as compared to usual physiotherapy (many different kinds of general exercises according to the preferences of the single therapists within different facilities).

\section{Authors' conclusions}

There is a lack of high quality evidence to recommend the use of SSE for AIS. One very low quality study suggested that these exercises may be more effective than electrostimulation, traction and postural training to avoid scoliosis progression, but better quality research needs to be conducted before the use of SSE can be recommended in clinical practice.

\section{PLAIN LANGUAGE SUMMARY}

\section{Exercises for scoliosis in teens}

Adolescent idiopathic scoliosis (AIS) is a rare (2\% to $3 \%$ of the general population) spinal deformity affecting young people aged 10 through the end of the growth period. The deformity may continue into adulthood. AIS is characterised by one or more threedimensional spinal curves. Disability, cosmetic deformity, pain, activity limitation, quality of life issues, breathing problems and the possibility of the scoliosis remaining with the person into and throughout adulthood are commonly associated with this condition. The cause of AIS is unknown.

Treatment for AIS varies according to the degree of severity of the curves. Just the same, exercise is almost always a part of the treatment plan. In milder cases, exercise may be the main treatment, and in more severe cases it may serve as an adjunct. In the UK and the US, physical therapy for scoliosis consists mainly of general strengthening and stretching exercises, along with exercise protocols with which the treating therapist is familiar. There is a corresponding feeling among practitioners in these geographical locations that physical therapy for scoliosis is not effective.

Scoliosis specific exercises (SSEs) are individualised exercises aimed at reducing the deformity. SSEs are taught in clinics that specialize in scoliosis. The exercises work by changing the soft tissue that affects the spine. SSEs are also thought to work by altering control of spinal movement. There are no known side effects or risks to using SSEs .

The purpose of this review was to evaluate the effectiveness of SSEs in reducing curve progression and postponing or avoiding invasive treatment such as surgery in adolescents with AIS. Two studies involving 154 patients total were included. The review found no evidences for or against SSE. The two included studies yielded very low quality evidence that SSEs added to other treatments are more effective than electrical stimulation, traction and posture training for avoiding curve progression, and that SSEs as a standalone treatment yield almost the same results as general physiotherapy.

Possible limitations of this review included the small number of studies that met the inclusion criteria and a high risk of bias, particularly selection bias. More randomised controlled trials are needed in this area, along with a deeper understanding of the types of SSEs useful for the adolescent with AIS. 
Patient or population: Adolescents with idiopathic scoliosis

Settings: Community

Intervention: Exercise plus other conservative treatments

Comparison: Other conservative treatments

\begin{tabular}{|c|c|c|c|}
\hline Outcomes & $\begin{array}{l}\text { Relative effect } \\
(95 \% \text { CI) }\end{array}$ & $\begin{array}{l}\text { No of Participants } \\
\text { (studies) }\end{array}$ & $\begin{array}{l}\text { Quality of the evidence } \\
\text { (GRADE) }\end{array}$ \\
\hline $\begin{array}{l}\text { Progression of scoliosis (in de- } \\
\text { grees assessed by x-ray) }\end{array}$ & $\begin{array}{l}\text { Thoracic curve: MD } 9.0095 \% \\
\text { confidence interval (Cl } 5.47 \\
\text { to12.53). Decrease in favour of } \\
\text { the exercise group. Effect clini- } \\
\text { cally relevant } \\
\text { Lumbar curve: MD } 8.0095 \% \\
\text { (Cl } 5.08 \text { to10.92). Decrease in } \\
\text { favour of the exercise group. Ef- } \\
\text { fect clinically relevant }\end{array}$ & $\begin{array}{l}80 \\
\text { (1 study) }\end{array}$ & $\begin{array}{l}\oplus \oplus \oplus \bigcirc \\
\text { low }\end{array}$ \\
\hline Cosmetic issues & Not measured & & \\
\hline Quality of life & Not measured & & \\
\hline Back pain & Not measured & & \\
\hline Psychological issues & Not measured & & \\
\hline Adverse effects & Not measured & & \\
\hline
\end{tabular}

GRADE Working Group grades of evidence

High quality: Further research is very unlikely to change our confidence in the estimate of effect.

Moderate quality: Further research is likely to have an important impact on our confidence in the estimate of effect and may change the estimate.

Low quality: Further research is very likely to have an important impact on our confidence in the estimate of effect and is likely to change the estimate.

Very low quality: We are very uncertain about the estimate.

\section{B A C K G ROU N D}

\section{Description of the condition}

Scoliosis is a complex deformity of the spine that develops in threedimensions and results in the appearance of frontal curves, fixed vertebral rotations, and a flattening of the sagittal physiological curves. When scoliosis develops between 10 years of age to the end of growth, it is called adolescent idiopathic scoliosis (AIS); idiopathic meaning that there is no known cause. A curvature in the spine can develop at any level of the spine and depending on the

Exercises for adolescent idiopathic scoliosis (Review)

Copyright () 2012 The Cochrane Collaboration. Published by John Wiley \& Sons, Ltd. 
vertebrae that are affected, is referred to as either a thoracic, thoracolumbar or lumbar scoliosis. While scoliosis can be secondary to other pathologies, in $70 \%$ to $80 \%$ of cases, the causes are unknown (SRS 2007). Adolescent idiopathic scoliosis is the most common diagnosis. The magnitude of scoliotic curves in the frontal plane is generally measured from $\mathrm{x}$-ray and is referred to as the Cobb angle (Weinstein 1983), named after the spinal surgeon who devised the method. The Cobb angle is the angle that measures the curvature of the spine in the frontal plane and measures the angle that includes all of the deformed vertebrae. It is generally agreed that curves that measure up to $25^{\circ} \mathrm{Cobb}$ are classed as mild curves; whereas moderate curves are considered to be those measuring from $25^{\circ}$ to $45^{\circ} \mathrm{Cobb}$ and severe curves measure over $45^{\circ} \mathrm{Cobb}$ angle. If scoliosis surpasses a critical threshold, usually considered to be $30^{\circ} \mathrm{Cobb}$, at the end of growth, the risk of health problems in adulthood increases significantly (Lonstein 2006). Problems include a decrease in the quality of life, disability, pain, increasing cosmetic deformity, functional limitations, sometimes pulmonary problems and possible progression during adulthood (Weinstein 2003). The Cobb angle is a measurement on the frontal plane only. Even if attempts have been made to have a more three-dimensional evaluation (2011 SOSORT Guidelines), today the gold standard remains the Cobb angle. To overcome this limitation, at the start and end of treatment, a complete radiographic evaluation is usually made, involving the assessment of spinal misalignment in the sagittal plane (the magnitude of lumbar lordosis and thoracic kyphosis are usually smaller than the physiological values) (2011 SOSORT Guidelines).

On the horizontal plane, the measurement of vertebral torsion is carried out with the Perdiolle torsiometer (Omerog lu 1996) or the Raimondi torsiometer (Weiss 1995b). Depending on the age of the individual at diagnosis, scoliosis evolves differently. According to the Scoliosis Research Society (SRS), the prevalence of AIS is $2 \%$ to $3 \%$ in the general population, almost $10 \%$ of whom require some form of treatment and up to $0.1 \%$ of whom will require surgery (Lonstein 2006). Adolescent idiopathic scoliosis is more commonly found in females (female:male ratio is around 7:1). Except for extreme cases, AIS does not typically cause any health problems during growth; however, the resulting surface deformity frequently has a negative impact on adolescents that can give rise to quality of life (QoL) issues and in the worst cases, psychological disturbances (Reichel 2003).

\section{Description of the intervention}

Due to the progressive nature of the deformity, adolescent patients are generally treated when the curvature is diagnosed. Furthermore, once the curve progresses, there are no treatments that succeed in fully correcting the spine. Depending on the mobility of the spine, reduction of the deformity can be difficult. The main treatment options for the prevention of scoliosis progression include scoliosis-specific exercises (SSE) and other forms of physical therapy, bracing and surgery (Lenssink 2005). The use of exercise for the treatment of AIS is controversial. Whilst it is routinely used in France, Germany, Italy, and a number of other countries in continental Europe, most centres in the UK and USA do not advocate its use. Most clinicians (both physiotherapists and surgeons) in the UK and USA do not normally appreciate the difference between SSE and general physiotherapy. Scoliosis-specific exercises consist of individually adapted exercises that are taught to the patients in a centre that is dedicated to scoliosis treatment. The patients learn an exercise protocol that is personalised according to medical and physiotherapeutic evaluations. Scoliosis-specific exercises include a series of specific physical movements performed with a therapeutic aim of reducing the deformity. Exercises work mechanically by changing the musculature and other soft tissues of the spine. It is also believed that SSE can alter the motor control of the spine by affecting neurological changes that interact with each other (Hawes 2003). On the other hand, generalised physiotherapy (GPT) is more generic, usually consisting of low-impact stretching and strengthening activities like Yoga, Pilates or Tai chi (taiji), but can include different exercise protocols according to the preferences of the therapist. The understanding within the generalised AIS treating community in the UK and USA may be based on the effectiveness of generalised physiotherapy, which has not been shown to be effective (2011 SOSORT Guidelines; Negrini 2008a).

The overall aim of SSE is to reduce the progression of the scoliotic deformity and the postponement and possible avoidance of brace prescription. Negrini 2008b and Ducongé 2002 reported that SSE can stabilize and reduce curve magnitude as well as improve respiratory function that may be altered by chest deformity. Exercise has also been reported to reduce the incidence of surgery (Weiss 2003b).

\section{How the intervention might work}

Scoliosis specific exercises can be used in three main clinical scenarios: (I) the sole use of exercise as the primary treatment of AIS for mild curves, (II) in conjunction with braces for moderate curves, and (III) during adulthood if the scoliosis curves exceed certain thresholds.

In the treatment of mild scoliosis of less than $25^{\circ} \mathrm{Cobb}$, intense three dimensional spine and rib-cage specific exercises are used in order to try and avoid the use of a brace. This critical Cobb angle is generally regarded as the threshold for brace prescription (Lonstein 2006; Weiss 2006b). In mild scoliosis cases where exercise is prescribed, SSE is predominantly used according to the recommendations made by the Study group on Scoliosis and Orthopaedic and Rehabilitative Treatment (SOSORT). The key objectives of physical exercise in mild cases of AIS are the stabilisation of the spine combined with three dimensional auto correction of the spine, pelvis and rib-cage. 
Several studies have also shown that bracing (which "binds" the thorax for continuous periods of time) tends to reduce the quality of life of young patients (Kotwicki 2007). Therefore, SSE can help to improve patients' quality of life by keeping the curve and rib hump under control for as long as possible, thus reducing the need for braces.

The second main clinical scenario for SSE use is in conjunction with brace treatment. In this case, the aims are to reduce the side effects of wearing a brace (muscle weakness, rigidity, flat back) and to improve the efficacy of internal brace pads (Romano 2006). SSE can also be used before a brace is worn to reduce spinal stiffness and improve mobility, thus helping to achieve a better correction (Negrini 2006). Moreover, SSE can help in avoiding losing correction while wearing the brace (Zaina 2009).

Finally, the third possible clinical scenario is during adulthood. If scoliosis exceeds certain thresholds, significant problems such as back pain, breathing dysfunction, contractures and progressive deformity can develop. These impairments and consequent disability can be addressed through exercise (Mamyama 2002).

\section{Why it is important to do this review}

A scoping literature search identified three systematic reviews on the topic, none of which followed Cochrane methodology (Lenssink 2005; Negrini 2003; Negrini 2008b). Therefore, we examined evidence that was published in these reviews and followed a more rigorous methodology to answer our clinical question "Is scoliosis-specific exercise therapy effective in delaying the progression of, or reducing the speed at which the curve progresses?": Preventing the progression of the disease means avoiding the need for bracing, surgery, or both. We did not include studies on bracing, because there is another review where this is covered (Negrini 2007). However, we considered all studies investigating the effects of SSEs added to bracing if compared with bracing alone.

\section{O B J E C T I VES}

The primary aim of this review was to evaluate the efficacy of scoliosis-specific exercises in the treatment of adolescent idiopathic scoliosis.

\section{METHODS}

\section{Criteria for considering studies for this review}

\author{
Types of studies
}

Randomised controlled trials (RCTs), quasi-RCTs (QRCTs) and observational studies were included, because it was anticipated that very few RCTs would be identified.

\section{Types of participants}

We included studies in which all patients were diagnosed as having AIS with at least a $10^{\circ} \mathrm{Cobb}$ angle, and were between the ages of 10 years and the end of bone growth (in female adolescents, this is approximately between the ages of 15 and 17 years; in male adolescents, this usually occurs between 16 and 19 years of age). The end of bone growth can be determined by the Risser sign, which quantifies the ossification of the iliac crest. Stage 4 indicates total ossification of the apophysis, while Stage 5, indicates fusion of the apophysis to the iliac crest and the end of further growth. The Greulich-Pyle atlas calculates the maturity of bones by assessing $\mathrm{x}$-rays of the left hand.

We excluded studies in which patients presented with any type of secondary scoliosis (congenital, neurological, metabolic, posttraumatic, etc), diagnosed according to the SRS criteria (SRS 2006).

\section{Types of interventions}

\section{Experimental intervention}

The experimental interventions in this review included all types of SSEs, which are considered to be "specific movements performed with a therapeutic aim of reducing the deformity". Sports, active recreational activities and generalised physiotherapy were not considered to be specific exercises for the treatment of scoliosis and studies including these types of activities were excluded.

\section{Comparison interventions}

Comparison interventions included no treatment; different types of SSEs, usual physiotherapy, doses or schedules of exercises; or other non-surgical treatments (e.g. braces, electrical stimulation, manual therapy).

Comparisons included: exercises versus no treatment, exercises plus another treatment versus the other treatment, exercises versus other treatments, exercises versus usual physiotherapy, different exercises versus each other, or different doses/schedules of exercises versus each other.

\section{Types of outcome measures}

This is a review of the effect of exercise on a radiological observation rather than a clinical syndrome. 


\section{Primary outcomes}

Progression of scoliosis, as measured by:

- Cobb angle in degrees (absolute values),

- angle of trunk rotation (ATR) in degrees (absolute values),

- number of patients who have progressed by more than $5^{\circ}$

Cobb,

- number of subjects for whom brace or surgery were

prescribed.

Cosmetic issues, as measured by:

- objective surface measurements, including Bunnel degrees

or other measurements with validated scales or questionnaires (such as the Walter Reed Visual Assessment Scale),

- topographic measurements e.g. the integrated shape imaging system (ISIS) angles, Quantec and Formetric (Rigo 2006).

Quality of life and disability, as measured by:

- specific validated questionnaires such as SRS-22, SF-36

(Asher 2003), BSSK, BrQ (Vasiliadis 2006).

Back pain, as measured by:

- visual analogue scale (VAS) or other validated measurement tools,

- use of drugs.

Psychological issues, as measured by:

- specific questionnaires such as sub-scales of SRS-22 and SF36, BrQ.

\section{Secondary outcomes}

Adverse effects, as outlined in identified trials, were also reported. All outcomes (primary and secondary) were measured in the very short-term (any result before the end of bone growth), the shortterm (results at the end of bone growth) and long-term (results in adulthood).

\section{Search methods for identification of studies}

\section{Electronic searches}

We searched the following electronic databases:

1) CENTRAL (The Cochrane Library to 30 March 2011), which includes the Cochrane Back Review Group Trials Register,

2) MEDLINE (1966 to 30 March 2011),

3) EMBASE (1980 to 30 March 2011),

4) CINAHL (1982 to 30 March 2011),

5) SportDiscus (1975 to 30 March 2011),

6) PsycInfo (1887 to 30 March 2011)

7) PEDro (1929 to 30 March 2011)
The updated search strategy recommended by the Cochrane Back Review Group for RCTs was used. This was adapted for cohort studies (Furlan 2009). The strategy includes subject headings $(\mathrm{MeSH})$ and text words. These include methodological terms, disorder terms and treatment terms, and are listed in full for MEDLINE, EMBASE, CINAHL and the other databases searched (Appendix 1; Appendix 2; Appendix 3; Appendix 4).

\section{Searching other resources}

The following strategies were also used:

1) screening of the reference lists of all relevant papers,

2) searching of the main electronic sources of ongoing trials $(\mathrm{Na}-$ tional Research Register, meta-Register of Controlled Trials; Clinical Trials),

3) searching of the Grey literature, including conference proceedings, $\mathrm{PhD}$ theses,

4) contacting investigators and authors in this field for information on unpublished or incomplete trials.

All searches included non-English language literature

\section{Data collection and analysis}

\section{Selection of studies}

Two review authors (SN, MR) independently screened the search results by reading titles and abstracts. Potentially relevant studies were obtained in full text and independently assessed for inclusion by two review authors, who resolved any disagreement through discussion. A third review author was contacted if disagreements persisted.

\section{Data extraction and management}

A standardised data extraction form was prepared and used to extract data from the included papers. Data extracted included study design (RCT, QRCT, prospective controlled cohort study), study characteristics (country, recruitment modality, study funding, risk of bias), patient characteristics (number of participants, age, sex, severity of scoliosis at baseline), description of the experimental and comparison interventions, co-interventions, adverse effects, duration of follow-up, outcomes assessed and results. Two review authors (SM and JB-S) who were not involved in the conduct of the primary studies, independently extracted the data. The data extraction form was not piloted because only two studies were included and data extracted were checked for any discrepancies for both included studies. Any disagreement was discussed and a third review author (TK) was consulted if disagreements persisted. Key findings were summarised in a narrative format and then assessed for inclusion in a meta-analysis where possible. 


\section{Assessment of risk of bias in included studies}

The risk of bias for RCTs and QRCTs was assessed using the 12 criteria recommended by the Cochrane Back Review Group (Furlan 2009), which are an expansion of the 'Risk of bias' criteria listed in The Handbook of Systematic Reviews of Interventions (Higgins 2009), and outlined in Appendix 5. The Newcastle-Ottawa Scale (NOS scale) (NOS 2000) was used to assess the observational studies. The NOS scale assesses three broad areas: selection bias, attrition bias, and detection bias. See Appendix 6 for details on 'Risk of bias' criteria for observational studies. Two review authors (SM and JB-S), who were not involved in the conduct of the primary studies, independently assessed the internal validity of the included studies. Any disagreement between the review authors was resolved by discussion; a third independent reviewer (NC) was consulted if disagreements persisted. 'Risk of bias' assessment was not blinded to trial authors, institution or journal since the review team is familiar with the literature.

The criteria recommended and defined by the Cochrane Back Review Group (Furlan 2009) were scored as 'yes', 'no' or 'unclear' and were reported in the 'Risk of bias' table. A trial with low risk of bias was defined as a trial that met, at a minimum, criteria A (randomisation), B (allocation concealment), C5 (outcome assessor blinding) and any three of the other criteria. It is very unlikely that trials on the effectiveness of exercises treatments could be blinded for participants and healthcare personnel. Nevertheless, the trials could have a blinded assessment of outcomes. The 'Risk of bias' tables were amended so they could be used to report the assessment of RCTs, QRCTs, and observational studies.

\section{Assessment of Clinical relevance}

Each trial was assessed by the review authors (MR, SN, FZ) for its clinical relevance, using the five questions outlined by Shekelle 1994 (Shekelle 1994; Appendix 7). All outcomes within each comparison were discussed. Clinical significance (Shekelle question 4) was defined as a $5^{\circ} \mathrm{Cobb}$ change, which is the reliability of radiographic examination and the international gold standard for minimally significant clinical change.

\section{Measures of treatment effect}

Dichotomous outcomes were analysed by SM (who was not involved in the conduct of the primary studies), by calculating the risk ratio (RR) for each trial, with the uncertainty in each result being expressed by $95 \%$ confidence intervals (CI). Continuous outcomes were analysed by calculating the mean difference (MD) or the standardised mean difference (SMD) with 95\% CI.

\section{Assessment of heterogeneity}

A P value of the $\mathrm{Chi}^{2}$ test less than 0.05 indicates a significant statistical heterogeneity. Clinical heterogeneity was also assessed for all retrieved studies. We planned to pool data only if the data were appropriately homogeneous.

\section{Data synthesis}

Meta-analysis was not performed because only one RCT and two prospective observational controlled trials were found, both of which reported on different aspects of the same study.

Despite the fact that there were insufficient data available to use quantitative analyses to summarise the data, we assessed the overall quality of the evidence for each primary outcome. To accomplish this, we used an adapted GRADE approach, as recommended by the Cochrane Back Review Group (Furlan 2009). The quality of the evidence on a specific outcome is based on the performance against six factors: study design, risk of bias, consistency and directness of results, precision of the data and non-biased reporting of the results across all studies that measured that particular outcome. The quality started at high when RCTs with a low risk of bias provided results for the outcome, and reduced by a level for each of the factors not met. For evidence that is provided by non-randomised trials, the quality started at low and is either reduced, based on performance against the same factors listed above (without study design) or increased if the evidence shows strong evidence of association, strong evidence of dose-response or evidence that all plausible confounders would have reduced the effect (GRADE 2004).

High quality evidence $=$ there are consistent findings among at least two RCTs with low risk of bias that are generalisable to the population in question. There were sufficient data, with narrow confidence intervals. There are no known or suspected reporting biases. Consistency is defined as $75 \%$ or more of the studies with similar results. Further research is very unlikely to change our confidence in the estimate of effect.

Moderate quality evidence $=$ one of the factors is not met. Further research is likely to have an important impact on our confidence in the estimate of effect and may change the estimate.

Low quality evidence $=$ two of the factors are not met. Further research is very likely to have an important impact on our confidence in the estimate of effect and is likely to change the estimate. Very low quality evidence $=$ three of the factors are not met. Any estimate of effect is very uncertain.

No evidence $=$ no evidence from RCTs

\section{Subgroup analysis and investigation of heterogeneity}

We had planned a subgroup analysis to explore the effects of the following variables: age, bone age, Cobb degrees and type of exercise in the case of significant statistical heterogeneity, but metaanalysis was not performed.

Comparison between primary and secondary analysis 
Separate analyses were performed for randomised (primary analysis) and observational studies (secondary analysis). Results obtained from the two analyses were compared and contrasted. Results of observational studies were added to the GRADE analysis as part of the comparison.

\section{Sensitivity analysis}

To incorporate the 'Risk of bias' assessment in the review process, stratification of intervention effects had initially been planned. This would have included estimates by risk of bias as sensitivity analyses, excluding studies with high risk of bias from the analysis if differences in results were seen among studies at different risks of bias. As a meta-analysis was not performed, a 'Risk of bias' assessment could not be conducted.

\section{RE S U L T S}

\section{Description of studies}

See: Characteristics of included studies; Characteristics of excluded studies.

\section{Results of the search}

With the bibliographic search, we identified 6807 references. After excluding duplicates we identified 6581 potentially relevant references; 6561 were excluded on the basis of title and abstracts, leaving 20 studies which were acquired in full text for further evaluation. See study flow diagram (Figure 1). 
Figure I. Study flow diagram.

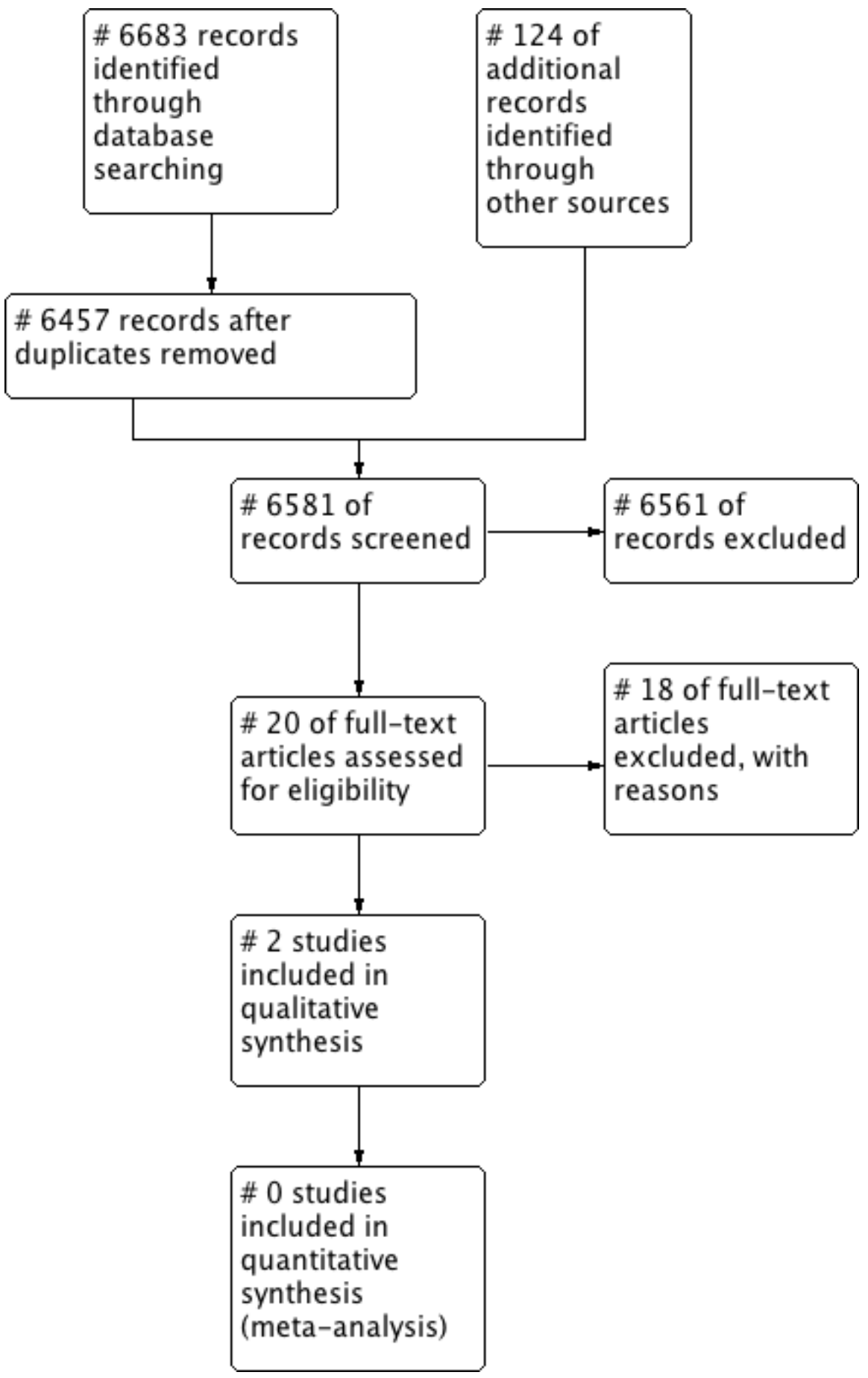


For substantive descriptions of the studies see Characteristics of included studies and Characteristics of excluded studies tables.

\section{Included studies}

We included two studies: one randomised controlled trial (Wan 2005) and one prospective controlled cohort study (Negrini 2008a).

The randomised trial by Wan 2005, included 80 adolescents. Electro-stimulation on the lateral body surface, traction therapy, postural training and postural advice during normal activities were prescribed to both groups. The experimental group also performed SSE.

The Negrini 2008a study of 74 adolescents prescribed the SEAS (Scientific Exercise Approah to Scoliosis) exercises (a type of SSE), which consisted of an individual education session of scoliosis- specific SEAS exercises to be performed every three months. SSEs were then performed at home two to three time per week. The control group performed usual physiotherapy, which included exercise protocols according to the preferences of their single therapist.

\section{Excluded studies}

Eighteen studies were excluded for the following reasons: 12 studies were excluded because of the study design, three because of outcome measures and three because of the type of intervention.

\section{Risk of bias in included studies}

Overall, the risk of bias in the included studies was very high. See Figure 2. 
Figure 2. 'Risk of bias' summary: review authors' judgements about each risk of bias item for each included study.

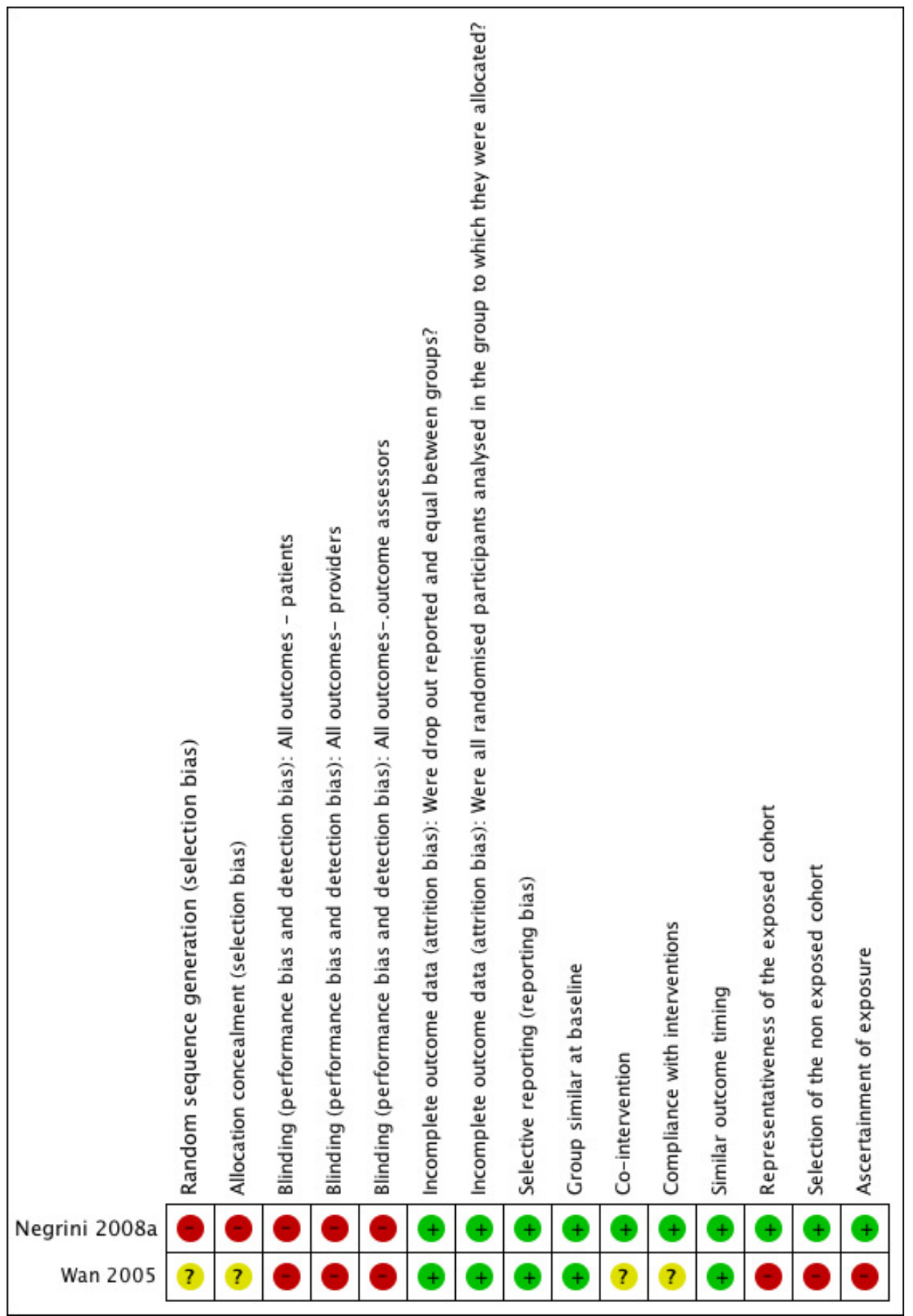




\section{Allocation}

Only one RCT was retrieved. The method used for random sequence generation and for concealment of allocation was not reported.

\section{Blinding}

Neither the RCT nor the observational prospective study could be blinded for patients and providers because of the kind of intervention assessed (exercises). The outcome assessor was not blinded in either study.

\section{Incomplete outcome data}

There were no drop-outs from the RCT; in the study by Negrini 2008 a the drop-out rate was $6.7 \%$ and was balanced across groups; these results were included in a worst-case analysis.

\section{Selective reporting}

All studies appeared to be free of selective reporting.

\section{Other potential sources of bias}

Groups similar at baseline: groups were similar in both the RCT and the cohort studies for age, gender and Cobb angle. No other potential confounders were listed and no adjustment for the most important confounding factors was performed in the observational study.

Information on compliance and co-interventions were not reported in Wan 2005. Compliance was high (95\%) and co-interventions were similar across groups in Negrini 2008a.

The timing of outcome assessments was similar among groups in both the Wan 2005 and Negrini 2008a studies.

In the observational study, the exposed cohort was representative of the population with idiopathic scoliosis.

In Negrini 2008a, the main outcome of interest (percentages of braced patients) could have been influenced by the lack of blinding of the treating physician, who was responsible for brace prescription.

\section{Effects of interventions}

See: Summary of findings for the main comparison

\section{Exercises plus other treatments versus other treatments only}

Progression of scoliosis: Wan 2005
- Thoracic curve: mean difference (MD) 9.00, (95\% confidence interval (CI) 5.47 to12.53). Statistically significant decrease in favour of the exercise group

- Lumbar curve: MD 8.00, (95\% CI 5.08 to10.92). Statistically significant decrease in favour of the exercise group.

There was no evidence for patient-related outcomes of cosmetic improvement, general improvement, disability or back pain.

\section{Different kind of exercises versus each other}

Progression of scoliosis: Negrini 2008a

Considering the per protocol analysis, the risk ratio (RR) for brace prescription was 0.24 , (95\% CI 0.06 to 1.04$)$. For the intentionto-treat analysis: RR 0.37 , (95\% CI 0.13 to 1.05 ).

In terms of Cobb angle degree, the RR for patients' improvement was 2.23 , (95\% CI 0.73 to 6.76 ); the RR for patients getting worse was 0.89 , (95\% CI 0.26 to 3.06). The RR for patient stability was 0.85 , (95\% CI 0.64 to 1.15$)$. The differences were not statically significant.

In regard to the angle of trunk rotation (ATR), the RR for improvement was 3.34, (95\% CI 0.36 to 30.68 ), for patients getting worse 0.56 , (95\% CI 0.21 to 1.47 ), for stability 1.11 , (95\% CI 0.85 to 1.47$)$. The differences were not statically significant.

The quality of evidence concerning the use of scoliosis-specific exercises (SSE) to reduce progression of scoliosis is very low. There are no studies on the efficacy of SSE to improve cosmetic issues, quality of life and disability, back pain and psychological issues.

\section{I S C USSION}

\section{Summary of main results}

Despite a comprehensive search of published and unpublished literature, we found only two studies that met the strict inclusion criteria. There was very low quality evidence from two studies (Negrini 2008a; Wan 2005) indicating that SSEs added to other treatments are more effective than electrostimulation, traction and postural training to avoid scoliosis progression, and that SSEs alone has almost similar results to usual physiotherapy. No data were found regarding the patient-centred outcomes of quality of life, back pain, psychological and cosmetic issues.

\section{Overall completeness and applicability of evidence}

Exercises for adolescent idiopathic scoliosis (Review) 
According to the evidence included in this review, the main finding is that there appears to be no evidence for or against exercises. The article by Wan et al did not provide enough details to replicate his protocol (Wan 2005). Conversely, Negrini described the intervention protocol in great detail, however, no details are given regarding "usual physiotherapy" because even the author was unaware of what was going on in the control group since it was quite heterogeneous and managed by independent therapists Negrini 2008a.

Out of the outcome measures chosen for this review, only data about radiological findings (Cobb angles) measuring curve magnitude and progression rate were available. This is one of the objectives of exercise, and it is relevant for patients, since curve progression can increase the risk of more aggressive treatments like bracing. Usually, progression is measured in terms of Cobb degrees; despite being the most used measure, this is a surrogate endpoint, since it is an indirect measure of the risk of future problems such as back pain, trunk decompensation and future progression during adulthood Weinstein 2003. Other possible measures of progression are ATR, and brace prescription rate that were used in the Negrini study. Brace prescription rate is a relevant outcome for patients because it is a more aggressive treatment Weiss 2006b. The limit of this outcome measure is that it is subjective, and potentially prone to bias. If the physician prescribing the brace is blinded to the study outcomes, these data are reliable, otherwise it can introduce a bias. This is exactly the same for surgery prescription/performance, which is one of the main outcomes for brace studies according to Scoliosis Resarch Society (SRS) criteria.

Other outcomes should be considered, mainly quality of life, cosmetic and psychological issues, since these are more relevant during adolescence than adulthood. Unfortunately, none of the papers meeting the inclusion criteria included these patient-centred outcomes within their studies.

\section{Clinical relevance}

This review suggests that to date, due to a lack of high quality RCTs in this area, there is no evidence for or against exercises, so hardly any clinical recommendations can be given.

As stated in the background to this review, the use of exercise for the treatment of adolescent idiopathic scoliosis (AIS) is controversial. Whilst it is currently routinely used in France, Germany, Italy, and a number of other countries in continental Europe, most centres in the UK and USA do not advocate its use. Until a high quality RCT is conducted, we will not know for certain whether SSE's are effective or not. A National Institute for Health Reseach (NIHR) Health Technology Assessment (HTA) feasibility study is currently being conducted in the UK. If the results of this study are positive then the first well conducted RCT can be performed and evidence found.

No statistically significant effects of SSE were found.
No major risks of the intervention have been reported in the literature, and no side effects were cited in the considered studies.

\section{Quality of the evidence}

There is no evidence for or against the use of SSE for treating idiopathic scoliosis. Moreover, it must be stressed that the results regarding brace prescription reported in Negrini 2008a were at high risk of detection bias because the physicians who prescribed the treatments, and who probably believed in their efficacy, were also the physicians who assessed the outcomes and decided whether or not braces should be prescribed or not.

\section{Potential biases in the review process}

The strength of the review is the extensive and comprehensive searches conducted, including a large number of different sources in many languages. The main weakness of the review is the absence of high quality studies in this field that make it impossible to reach any firm conclusions.

\section{Agreements and disagreements with other studies or reviews}

In three previous reviews conducted on the effectiveness of scoliosis-specific exercises (Lenssink 2005; Negrini 2003; Negrini 2008b), a greater number of studies of lower methodological quality were included: although the quality of the available studies was low, results were consistent in favour of the efficacy of SSE. In this review, as it was necessary to limit the search to high quality studies, these results could not be confirmed

\section{AUTHORS, CONCLUSIONS}

\section{Implications for practice}

There is lack of high quality evidence to recommend the use of scoliosis-specific exercises for adolescent idiopathic scoliosis. One very low quality study (Wan 2005) suggested that these exercises may be more effective than electrostimulation, traction and postural training to avoid scoliosis progression, but better quality research needs to be conducted before the use of scoliosis-specific exercises can be recommended in clinical practice.

\section{Implications for research}

More randomised controlled trials are needed to clarify the real role of scoliosis-specific exercises as a treatment-modality for mild to moderate adolescent idiopathic scoliosis compared with no treatment. In addition to this overriding goal, further research should 
also endeavour to clearly define the best types of scoliosis-specific exercises for different curve types as well as the most effective methods (frequency and intensity) among those available. In order to achieve this, multicentre studies carried out by key international research centres on matched groups of scoliosis patients need to be conducted.

\section{ACKNOWLEDGEMENTS}

We would like to thank Rachel Couban, Trials Search Co-ordinator of the Cochrane Back Review Group for her help with the search strategies.

\section{R E F E R E N C E S}

\section{References to studies included in this review}

Negrini 2008a \{published data only\}

Negrini S, Negrini A, Romano M, Verzini N, Negrini Al, Parzini S. A controlled prospective studiY on the efficacy of SEAS.02 exercises in preventing progression and braces in mild idiopathic scoliosis. Studies in Health Technology and Informatics 2006;123:523-6.

* Negrini S, Zaina F, Romano M, Negrini A, Parzini S. Specific exercises reduce brace prescription in adolescent idiopathic scoliosis: A prospective controlled cohort studies with worst case analysis. Journal of Rehabilitation Medicine 2008;40:451-5.

Wan 2005 \{published data only\}

Wan Li, Wang Guo-Xin, Bian Rong. Results of exercise therapy in treatment of essentially S-shaped scoliosis patients: Evaluations of Cobbs angle in the breast and lumbar segment. Chinese Journal of Clinical Rehabilitation 2005;9(34):82-4.

\section{References to studies excluded from this review}

Alves 2006 \{published data only\}

Alves VLDS, Stirbulov R, Avanzi O. Impact of a physical rehabilitation program on the respiratory function of adolescents with idiopathic scoliosis. Chest 2006;130(2): $500-5$.

Athanasopoulos 1999 \{published data only\} Athanasopoulos S, Paxinos T, Tsafantakis E, Zachariou K, Chatziconstantinou S. The effect of aerobic training in girls with idiopathic scoliosis. Scandinavian Journal of Medicine $\&$ Science in Sports 1999;9(1):36-40.

Carman 1985 \{published data only\} Carman D, Roach JW, Speck G, Wenger DR, Herring JA. Role of exercises in the Milwaukee brace treatment of scoliosis. Journal of Pediatric Orthopedics 1985;5(1):65-8.
Durmala 2002 \{published data only\}

Durmala J, Dobosiewicz K, Jendrzejek H, Pius W. Exercise efficiency of girls with idiopathic scoliosis based on the ventilatory anaerobic threshold. Studies in Health Technology \& Informatics 2002;91:357-60.

Durmala 2003 \{published data only\}

Durmala J, Dobosiewicz K, Kotwicki T, Jendrzejek H. Influence of asymmetric mobilisation of the trunk on the Cobb angle and rotation in idiopathic scoliosis in children and adolescents. Ortopedia Traumatologia Rehabilitacja 2003;5(1):80-5.

Dyner-Jama 2000 \{published data only\}

Dyner-Jama I, Dobosiewicz K, Niepsuj K, Niepsuj G, Jedrzejewska A, Czernicki K. Effect of asymmetric respiratory exercise therapy on respiratory system function; evaluation using spirometric examination in children with idiopathic scoliosis. Wiadomosci Lekarskie 2000;53(11-12): 603-10.

Kowalski 2001 \{published data only\}

Kowalski IM, Protasiewicz H. An authorial modification of kinesitherapy in idiopathic scoliosis. Ortopedia, Traumatologia, Rehabilitacja 2001;3(2):276-81.

Mamyama 2002 \{published data only\}

Mamyama T, Kitagawal T, Takeshita K, Nakainura K. Side shift exercise for idiopathic scoliosis after skeletal maturity. Studies in Health Technology \& Informatics 2002;91:361-4.

Maruyama 2003 \{published data only\}

Maruyama T, Kitagawa T, Takeshita K, Mochizuki K, Nakamura K. Conservative treatment for adolescent idiopathic scoliosis: can it reduce the incidence of surgical treatment?. Pediatric Rehabilitation 2003;6(3-4):215-9.

McIntire 2008 \{published data only\} McIntire KL, Asher MA, Burton DC, Liu W. Treatment of adolescent idiopathic scoliosis with quantified trunk rotational strength training: a pilot study. Journal of Spinal Disorders \& Techniques 2008;21(5):349-58. 
Mooney 2003 \{published data only\}

Mooney V, Brigham A. The role of measured resistance exercises in adolescent scoliosis. Orthopedics 2003;26(2): $167-71$.

Weiss 1991 \{published data only\}

Weiss HR. The effect of an exercise program on vital capacity and rib mobility in patients with idiopathic scoliosis. Spine 1991;16(1):88-93.

Weiss 1992 \{published data only\}

Weiss HR. The progression of idiopathic scoliosis under the influence of a physiotherapy rehabilitation programme. Physiotherapy 1992;78(11):815-21.

Weiss 1995a \{published data only\}

Weiss HR. The Schroth scoliosis-specific back school-initial results of a prospective follow-up study. Zeitschrift fur Orthopadie und Ihre Grenzgebiete 1995;133(2):114-7.

Weiss 1997 \{published data only\}

Weiss HR, Lohschmidt K, el Obeidi N, Verres C. Preliminary results and worst-case analysis of in patient scoliosis rehabilitation. Pediatric Rehabilitation 1997;1(1): $35-40$.

Weiss 2002 \{published data only\}

Weiss HR, Heckel I, Stephan C. Application of passive transverse forces in the rehabilitation of spinal deformities: A randomized controlled study. Studies in Health Technology \& Informatics 2002;88:304-8.

Weiss 2003a \{published data only\}

Weiss HR, Weiss G, Petermann F. Incidence of curvature progression in idiopathic scoliosis patients treated with scoliosis in-patient rehabilitation (SIR): an aged-and sexmatched controlled study. Pediatric Rehabilitation 2003;6 (1):23-30.

Weiss 2006a \{published data only\}

Weiss HR, Klein R. Improving excellence in scoliosis rehabilitation: a controlled study of matched pairs. Pediatric Rehabilitation 2006;9(3):190-200.

\section{Additional references}

\section{SOSORT Guidelines}

Negrini S, Aulisa AG, Aulisa L, Circo AB, de Mauroy JC, Durmala J, et al.2011 SOSORT Guidelines: Orthopaedic and Rehabilitation Treatment of Idiopathic Scoliosis During Growth. Scoliosis 2012 Jan 20;7(1):3.

Asher 2003

Asher M. The reliability and concurrent validity of the Scoliosis Research Society 22-patient questionnaire for idiopathic scoliosis. Spine 2003;28(1):63-9.

Boutron 2005

Boutron I, Moher D, Tugwell P, Giraudeau B, Poiraudeau $S$, Nizard R, et al.A checklist to evaluate a report of a non pharmacological trial (CLEAR NPT) was developed using consensus. Journal of Clinical Epidemiology 2005;58 1233-40.
Ducongé 2002

Ducongé PL. [La rééducation de la scoliose. Mythe ou réalitè?]. Résonances Européennes du Rachis 2002;10: 1229-36.

Furlan 2009

Furlan AD, Pennick V, Bombardier C, van Tulder M, Editorial Board of the Cochrane Back Review Group. 2009 Updated method guidelines for systematic reviews in the Cochrane Back Review Group. Spine 2009;34(18): 1929-41.

GRADE 2004

The GRADE Working Group. Grading quality of evidence and strength of recommendations. BMJ 2004 (Published 17 June 2004);328:1490. [DOI: 10.1136/ bmj.328.7454.1490]

Hawes 2003

Hawes MC. The use of exercises in the treatment of scoliosis: an evidence-based critical review of the literature. Pediatric Rehabilitation 2003 Jul-Dec;6(3-4):171-82.

Higgins 2009

Higgins JPT, Green S (Editors). Cochrane Handbook for Systematic Reviews of Interventions Version 5.0.2 [updated September 2009]. The Cochrane Collaboration; Available from www.cochrane-handbook.org 2009.

Kotwicki 2007

Kotwicki T, Kinel E, Stry W, Szulc A. Estimation of the stress related to conservative scoliosis therapy: an analysis based on BSSQ questionnaires. Scoliosis 2007;3(2):1.

\section{Lenssink 2005}

Lenssinck ML, Frijlink AC, Berger MY, Bierman-Zeinstra SM, Verkerk K, Verhagen AP. Effect of bracing and other conservative interventions in the treatment of idiopathic scoliosis in adolescents: A systematic review of clinical trials. Physical Therapy 2005;85(12):1329-39.

Lonstein 2006

Lonstein JE. Scoliosis: Surgical versus non-surgical treatment. Clinical Orthopaedics and Related Research 2006; 443:284-59.

\section{Negrini 2003}

Negrini S, Antonini G, Carabalona R, Minozzi S. Physical exercises as a treatment for adolescent idiopathic scoliosis. A systematic review. Pediatric Rehabilitation 2003 Jul-Dec; 6(3-4):227-35.

\section{Negrini 2006}

Negrini S, Negrini A, Romano M, Verzini N, Negrini A, Parzini S. A controlled prospective study on the efficacy of SEAS.02 exercises in preparation to bracing for idiopathic scoliosis. Studies in Health Technology \& Informatics 2006; 123:519-22.

Negrini 2007

Negrini S, Minozzi S, Bettany-Saltikov J, Zaina F, Chockalingam N, Grivas TB, et al.Braces for idiopathic scoliosis in adolescents. Cochrane Database of Systematic Reviews 2010, Issue 1. [DOI: 10.1002/ 14651858.CD006850.pub2] 
Negrini 2008b

Negrini S, Fusco C, Minozzi S, Atanasio S, Zaina F, Romano $M$. Exercises reduce the progression rate of adolescent idiopathic scoliosis: Results of a comprehensive systematic review of the literature. Disability and Rehabilitation 2008; 30(10):772-85.

\section{NOS 2000}

Wells GA, Shea B, O'Connell D, Peterson J, Welch V, Losos $\mathrm{M}$, et al.The Newcastle-Ottawa Scale (NOS) for assessing the quality of nonrandomised studies in meta-analyses. www.ohri.ca/programs/clinical 'epidemiology/oxford.htm (accessed December 2008) 2000.

\section{Omeroğ lu 1996}

Omeroğ lu H, Ozekin O, Biçimoğ lu A. Measurement of vertebral rotation in idiopathic scoliosis using the Perdriolle torsionmeter: a clinical study on intraobserver and interobserver error. European Spine Journal 1996;5(3): $167-71$.

\section{Reichel 2003}

Reichel D, Schanz J. Developmental psychological aspects of scoliosis treatment. Pediatric Rehabilitation 2003;6(3-4): $221-5$.

\section{Rigo 2006}

Rigo M, Quera-Salva G, Villagrasa M. Sagittal configuration of the spine in girls with idiopathic scoliosis: Progressing rather than initiating factor. Studies in Health Technology and Informatics 2006;123:90-4.

\section{Romano 2006}

Romano M, Carabalona S, Petrilli S, Sibilla P, Negrini S. Forces exerted during exercises by patients with adolescent idiopathic scoliosis wearing Fiberglass braces. Scoliosis 2006; 21:1-12.

\section{Shekelle 1994}

Shekelle PG, Andersson G, Bombardier C, Cherkin D, Deyo R, Keller R. A brief introduction to the critical reading of the clinical literature. Spine 1994;19:2028s-31s.

\section{SRS 2006}

Scoliosis Resarch Society. Brace Wear Compliance. http: //www.srs.org/professionals/bracing manuals/section3.pdf 2006.

\section{SRS 2007}

Scoliosis Research Society. The Scoliosis Research Society Brace Manual. Introduction. http://www.srs.org/ professionals/bracing manuals/section1.pdf 2007.

\section{van Tulder 2003}

van Tulder M, Furlan A, Bombardier C, Bouter L, Editorial Board Cochrane Back Review Group. Updated method guidelines for systemic reviews in the Cochrane Collaboration Back Review Group. Spine 2003;28(12): 1290-9.

\section{Vasiliadis 2006}

Vasiliadis E, Grivas TB, Gkoltsiou K. Development and preliminary validation of Brace Questionnaire (BrQ): a new instrument for measuring quality of life of brace treated scoliotics. Scoliosis 2006;1:7. [DOI: 10.1186/ 1748-7161-1-7]

\section{Weinstein 1983}

Weinstein SL, Ponseti IV. Curve progression in idiopathic scoliosis. Journal of Bone and Joint Surgery 1983;65(4): 447-55.

\section{Weinstein 2003}

Weinstein SL, Dolan LA, Spratt KF, Peterson KK, Spoonamore MJ, Ponseti IV. Health and function of patients with untreated idiopathic scoliosis: A 50-year natural history study. JAMA 2003;289(5):559-67.

\section{Weiss 1995b}

Weiss HR. Measurement of vertebral rotation: Perdriolle versus Raimondi. European Spine Journal 1995;4(1):34-8.

\section{Weiss 2003b}

Weiss HR, Weiss G, Schaar HJ. Incidence of surgery in conservatively treated patients with scoliosis. Pediatric Rehabilitation 2003;6:209-14.

\section{Weiss 2006b}

Weiss HR, Negrini S, Hawes MC, Rigo M, Kotwicki $\mathrm{T}$, Members of the SOSORT. Physical exercises in the treatment of idiopathic scoliosis at risk of brace treatment SOSORT consensus paper 2005. Scoliosis 2006;1:6.

\section{Zaina 2009}

Zaina F, Negrini S, Atanasio S, Fusco C, Romano M, Negrini A. Specific exercises performed in the period of brace weaning can avoid loss of correction in Adolescent Idiopathic Scoliosis (AIS) patients. Scoliosis 2009 Apr 7;4 (8).

* Indicates the major publication for the study 


\title{
CHARACTERISTICS OF STUDIES
}

\section{Characteristics of included studies [ordered by study ID]}

\author{
Negrini 2008a
}

Methods

Participants

Interventions

Outcomes

Progression of scoliosis as measured by Cobbs angle progression and ATR

Progression of scoliosis as measured by number of braces patients within one-year follow-

up

\section{Notes}

\section{Risk of bias}

\begin{tabular}{l|l|l}
\hline Bias & Authors' judgement & Support for judgement \\
\hline $\begin{array}{l}\text { Random sequence generation (selection } \\
\text { bias) }\end{array}$ & High risk & Prospective controlled cohort study. \\
\hline Allocation concealment (selection bias) & High risk & $\begin{array}{l}\text { Prospective controlled cohort study "The pa- } \\
\text { tients themselves decided whether they preferred } \\
\text { to be treated according to our exercise protocol }\end{array}$
\end{tabular}

Exercises for adolescent idiopathic scoliosis (Review)

Experimental: $\mathrm{N}=35$ : SEAS exercises according to the Italian Scientific Spine Institute (ISICO) approach;

The SEAS (Scientific Exercise Approah to Scoliosis) protocol consists of an individual education session at specialized ISICO Center (1.5 hours session every 2-3 months) and exercises are then performed by the patient twice a week at home or at a gym. Main elements of SEAS Approach are Active Self-Correction, a complex movement to obtain the best three dimensional alignment of scoliotic spine associated with "distracting" elements (imbalance, external weight, co-ordination task) for improvement of spine stabilisation and to obtain the neuromotor rehabilitation

Control: $\mathrm{N}=39$ : Usual physiotherapy group: many different exercise protocols at a local facility according to the preferences of their single therapist. In most cases the exercises were performed in a group context, while in all cases they lasted 45 to $90 \mathrm{~min}$ and were performed 2 or 3 times per week. In some cases, the patients were required to repeat their exercises daily at home 


\begin{tabular}{|c|c|c|}
\hline & & $\begin{array}{l}\text { (the SEAS group) or by a rehabilitation centre or } \\
\text { single physiotherapist of their choice (the usual } \\
\text { physiotherapy (UP) group). They were thus di- } \\
\text { vided into } 2 \text { groups through self-selection" }\end{array}$ \\
\hline $\begin{array}{l}\text { Blinding (performance bias and detection } \\
\text { bias) } \\
\text { All outcomes - patients }\end{array}$ & High risk & $\begin{array}{l}\text { Blinding of patients not possible for the kind of } \\
\text { intervention }\end{array}$ \\
\hline $\begin{array}{l}\text { Blinding (performance bias and detection } \\
\text { bias) } \\
\text { All outcomes- providers }\end{array}$ & High risk & $\begin{array}{l}\text { Blinding of providers not possible for the kind of } \\
\text { intervention }\end{array}$ \\
\hline $\begin{array}{l}\text { Blinding (performance bias and detection } \\
\text { bias) } \\
\text { All outcomes-.outcome assessors }\end{array}$ & High risk & $\begin{array}{l}\text { "physicians were neutral observers because they } \\
\text { were not aware of the study being performed and } \\
\text { they were focused only on the patients' needs, } \\
\text { although they were not blinded to the treatment } \\
\text { applied" }\end{array}$ \\
\hline $\begin{array}{l}\text { Incomplete outcome data (attrition bias) } \\
\text { Were drop out reported and equal between } \\
\text { groups? }\end{array}$ & Low risk & $\begin{array}{l}\text { There were } 5 \text { drop-outs: } 2 \text { in the SEAS group and } \\
3 \text { in the UP group }\end{array}$ \\
\hline $\begin{array}{l}\text { Incomplete outcome data (attrition bias) } \\
\text { Were all randomised participants analysed } \\
\text { in the group to which they were allocated? }\end{array}$ & Low risk & $\begin{array}{l}\text { The } 5 \text { patients who dropped out were included } \\
\text { in the worst-case analysis }\end{array}$ \\
\hline Selective reporting (reporting bias) & Low risk & \\
\hline Group similar at baseline & Low risk & $\begin{array}{l}\text { No difference in mean age. No statistically signif- } \\
\text { icant difference was found between the } 2 \text { groups } \\
\text { at baseline for any of the scoliosis parameters }\end{array}$ \\
\hline Co-intervention & Low risk & $\begin{array}{l}\text { Patients were required to perform sport activities. } \\
\text { No other intervention was provided }\end{array}$ \\
\hline Compliance with interventions & Low risk & $\begin{array}{l}\text { "The number of sessions per week was } 2.0, \mathrm{~min} \\
\text { per session were } 48 \text {, and compliance rate } 95 \% \text {. } \\
\text { In addition, no differences were found between } \\
\text { the } 2 \text { groups with respect to these parameters" }\end{array}$ \\
\hline Similar outcome timing & Low risk & \\
\hline Representativeness of the exposed cohort & Low risk & $\begin{array}{l}\text { The sample is truly representative of the average } \\
\text { adolescent with scoliosis }\end{array}$ \\
\hline Selection of the non exposed cohort & Low risk & $\begin{array}{l}\text { The sample has been drawn from the same com- } \\
\text { munity as the exposed cohort }\end{array}$ \\
\hline
\end{tabular}




\section{Negrini 2008a (Continued)}

\begin{tabular}{|c|c|c|}
\hline Ascertainment of exposure & Low risk & Clinical records. \\
\hline \multicolumn{3}{|l|}{ Wan 2005} \\
\hline Methods & \multicolumn{2}{|c|}{ Randomised controlled trial. } \\
\hline Participants & \multicolumn{2}{|c|}{$\begin{array}{l}80 \text { patients with double curve (S-shaped) scoliosis. Mean age: } 15 \pm 4 \text {; female: } 43.50 \\
\text { double curves (right thoracic and left lumbar); } 30 \text { had left thoracic and right lumbar } \\
\text { curves } \\
\text { Exclusion criteria: single curve (C-shaped) scoliosis. } \\
\text { Mean Cobb angle at start was: thoracic } 25 \pm 13^{\circ} \text {, lumbar } 23 \pm 11^{\circ}\end{array}$} \\
\hline
\end{tabular}

Interventions

Experimental: $\mathrm{N}=40$ : The same as control plus gymnastic exercise for correction of essential S-shaped scoliosis. Exercises were performed in a lying or creeping position, once a day

control: $\mathrm{N}=40$ : Electro-stimulation on the lateral body surface by a therapeutic apparatus for correction of lateral curvature. The duration of therapy was increased gradually, beginning with three times a day for thirty minutes each. On the second day, it was twice for one hour each. On the third day it was once for three hours. Thereafter, treatment was increased by one hour every day until it reached eight hours per day. Subsequently, it progressed to traction therapy. When the curvature is pronounced in the upper body, mandibular traction is done using pelvic traction for obvious lateral curvature twice a day, with each session lasting thirty minutes. Both groups also underwent postural training during treatment. Patients were advised to maintain a straight, symmetrical posture during normal activities

Outcomes

Progression of scoliosis (Cobb's Angles in degrees assessed by X-Ray). Difference between baseline and six-month follow-up

Notes

\section{Risk of bias}

\begin{tabular}{|c|c|c|}
\hline Bias & Authors' judgement & Support for judgement \\
\hline $\begin{array}{l}\text { Random sequence generation (selection } \\
\text { bias) }\end{array}$ & Unclear risk & "patients were randomly divided into two groups" \\
\hline Allocation concealment (selection bias) & Unclear risk & "patients were randomly divided into two groups" \\
\hline $\begin{array}{l}\text { Blinding (performance bias and detection } \\
\text { bias) } \\
\text { All outcomes - patients }\end{array}$ & High risk & $\begin{array}{l}\text { Blinding of patients not possible for the kind of inter- } \\
\text { vention }\end{array}$ \\
\hline
\end{tabular}

Blinding (performance bias and detection High risk bias) Blinding of providers not possible for the kind of interAll outcomes- providers vention 
Wan 2005 (Continued)

\begin{tabular}{|c|c|c|}
\hline $\begin{array}{l}\text { Blinding (performance bias and detection } \\
\text { bias) } \\
\text { All outcomes-.outcome assessors }\end{array}$ & High risk & $\begin{array}{l}\text { "The planning, execution and evaluation were all car- } \\
\text { ried out by the author; The first author used SPSS } 10 \text {. } \\
0 \text { statistical software to manage data. This was used to } \\
\text { compare before and after treatment in association with } \\
\text { testing }\end{array}$ \\
\hline $\begin{array}{l}\text { Incomplete outcome data (attrition bias) } \\
\text { Were drop out reported and equal between } \\
\text { groups? }\end{array}$ & Low risk & No drop-outs from the study. \\
\hline $\begin{array}{l}\text { Incomplete outcome data (attrition bias) } \\
\text { Were all randomised participants analysed } \\
\text { in the group to which they were allocated? }\end{array}$ & Low risk & \\
\hline Selective reporting (reporting bias) & Low risk & \\
\hline Group similar at baseline & Low risk & $\begin{array}{l}\text { Thoracic Cobb angle was } 25 \pm 13^{\circ} \text {, and the lumbar one } \\
\text { was } 23 \pm 11^{\circ} \text { in the control group } \\
\text { Thoracic Cobb angle was } 26 \pm 12^{\circ} \text { and the lumbar one } \\
\text { was } 24 \pm 10^{\circ} \text { in the experimental group }\end{array}$ \\
\hline Co-intervention & Unclear risk & Information not reported. \\
\hline Compliance with interventions & Unclear risk & Information not reported. \\
\hline Similar outcome timing & Low risk & \\
\hline Representativeness of the exposed cohort & High risk & Not assessed for RCT. \\
\hline Selection of the non exposed cohort & High risk & Not assessed for randomised controlled trial. \\
\hline Ascertainment of exposure & High risk & Not assessed for randomised controlled trial. \\
\hline
\end{tabular}

ATR: angle of trunk rotation

Characteristics of excluded studies [ordered by study ID]

\begin{tabular}{l|l}
\hline Study & Reason for exclusion \\
\hline Alves 2006 & Study design not in the inclusion criteria: uncontrolled study \\
\hline Athanasopoulos 1999 & Different outcome than described in inclusion criteria (parameters of pulmonary function)
\end{tabular}


(Continued)

\begin{tabular}{|c|c|}
\hline Carman 1985 & Patient in treatment group wore a brace. \\
\hline Durmala 2002 & Different outcome than described in inclusion criteria (ventilatory anaerobic threshold) \\
\hline Durmala 2003 & Study design not in the inclusion criteria: uncontrolled study \\
\hline Dyner-Jama 2000 & Different outcome than described in inclusion criteria (spirometric indexes) \\
\hline Kowalski 2001 & Study design not in the inclusion criteria: not reported if the study was prospective or retrospective \\
\hline Mamyama 2002 & Study design not in the inclusion criteria: uncontrolled study \\
\hline Maruyama 2003 & Study design not in the inclusion criteria: uncontrolled study \\
\hline McIntire 2008 & Study design not in the inclusion criteria: uncontrolled study \\
\hline Mooney 2003 & Study design not in the inclusion criteria: uncontrolled study \\
\hline Weiss 1991 & Study design not in the inclusion criteria: uncontrolled study \\
\hline Weiss 1992 & Study design not in the inclusion criteria: retrospective and uncontrolled study \\
\hline Weiss 1995 a & Study design not in the inclusion criteria: uncontrolled study \\
\hline Weiss 1997 & Study design not in the inclusion criteria: uncontrolled study \\
\hline Weiss 2002 & Use of passive corrective forces. \\
\hline Weiss 2003a & $\begin{array}{l}\text { Study design not in the inclusion criteria: results at follow-up of two different uncontrolled prospective case } \\
\text { series }\end{array}$ \\
\hline Weiss 2006a & Some patients in treatment group wore a brace. \\
\hline
\end{tabular}




\section{DATAANDANALYSES}

This review has no analyses.

\section{A P P E N D I C ES}

\section{Appendix I. MEDLINE search strategy}

1 randomized controlled trial.pt.

2 controlled clinical trial.pt.

3 randomized.ab.

4 placebo.ab,ti.

5 drug therapy.fs.

6 randomly.ab,ti.

7 trial.ab,ti.

8 groups.ab,ti.

9 or/ $1-8$

10 (animals not (humans and animals)).sh.

119 not 10

12 Comparative Study/

13 exp Evaluation Studies/

14 exp Follow-Up Studies/

15 exp Prospective Studies/

16 exp Cross-Over Studies/

17 exp Epidemiologic Studies/

18 exp Case-Control Studies/

19 exp Cohort Studies/

20 exp Cross-Sectional Studies/

21 (cohort adj (study or studies)).mp.

22 cohort analy\$.mp.

23 (follow up adj (study or studies)).mp.

24 (observational adj (study or studies)).mp.

25 longitudinal.mp.

26 retrospective.mp

27 cross sectional.mp.

28 control\$.mp.

29 prospective $\$ . m p$.

30 volunteer.mp.

31 or/ $12-30$

3211 or 31

33 exp Spinal Diseases/

34 exp Scoliosis/

35 scoliosis.mp.

36 or/33-35

37 exp Exercise/

38 exercis\$.mp.

39 exp Exercise Therapy/

40 exp Exercise Movement Techniques/

41 exp Physical Therapy Modalities/

Exercises for adolescent idiopathic scoliosis (Review)

Copyright $\odot 2012$ The Cochrane Collaboration. Published by John Wiley \& Sons, Ltd. 
42 physiotherap\$.mp.

43 exp Rehabilitation/

44 or/37-43

4532 and 36 and 44

\section{Appendix 2. EMBASE search strategy}

1 exp Clinical Study/

2 exp Case Control Study/

3 exp Family Study/

4 exp Longitudinal Study/

5 exp Retrospective Study/

6 exp Prospective Study/

7 exp Cohort Analysis/

8 (cohort adj (study or studies)).mp. [mp=title, abstract, subject headings, heading word, drug trade name, original title, device manufacturer, drug manufacturer name]

9 (case control adj (study or studies)).mp. [mp=title, abstract, subject headings, heading word, drug trade name, original title, device manufacturer, drug manufacturer name]

10 (follow up adj (study or studies)).mp. [mp=title, abstract, subject headings, heading word, drug trade name, original title, device manufacturer, drug manufacturer name]

11 (observational adj (study or studies)).mp. [mp=title, abstract, subject headings, heading word, drug trade name, original title, device manufacturer, drug manufacturer name]

12 (epidemiologic\$ adj (study or studies)).mp. [mp=title, abstract, subject headings, heading word, drug trade name, original title, device manufacturer, drug manufacturer name]

13 (cross sectional adj (study or studies)).mp. [mp=title, abstract, subject headings, heading word, drug trade name, original title, device manufacturer, drug manufacturer name]

14 exp Comparative Study/

15 evaluation study.mp.

16 follow-up study.mp. or exp Follow Up/

17 Crossover Procedure/

18 prospective\$.mp.

19 exp VOLUNTEER/

20 or/1-19

21 Clinical Article/

22 exp Clinical Study/

23 Clinical Trial/

24 Controlled Study/

25 Randomized Controlled Trial/

26 Major Clinical Study/

27 Double Blind Procedure/

28 Multicenter Study/

29 Single Blind Procedure/

30 Phase 3 Clinical Trial/

31 Phase 4 Clinical Trial/

32 crossover procedure/

33 placebo/

34 or/21-33

35 allocat\$.mp.

36 assign \$.mp.

37 blind \$.mp.

38 (clinic $\$$ adj25 (study or trial)).mp.

39 compar\$.mp.

Exercises for adolescent idiopathic scoliosis (Review)

Copyright $\odot 2012$ The Cochrane Collaboration. Published by John Wiley \& Sons, Ltd. 
40 control\$.mp.

41 cross?over.mp.

42 factorial\$.mp.

43 follow?up.mp.

44 placebo $\$ . \mathrm{mp}$.

45 prospectiv\$.mp.

46 random $\$ . \mathrm{mp}$.

47 ( (singl\$ or doubl\$ or trebl\$ or tripl\$) adj25 (blind\$ or mask\$)).mp.

48 trial.mp.

49 (versus or vs).mp.

50 or/35-49

5134 and 50

5220 or 51

53 Human/

54 Nonhuman/

55 exp ANIMAL/

56 Animal Experiment/

5754 or 55 or 56

5853 not 57

5952 not 57

6058 or 59

61 exp SPINE/

62 exp Spine Disease/

63 exp SCOLIOSIS/

64 exp Idiopathic Scoliosis/

65 scoliosis.mp.

66 or/61-65

67 exp Brace/

68 brace $\$ . m p$.

69 bracing.mp.

70 exp ORTHOTICS/

71 exp orthopedic equipment/

72 or/ $67-71$

73 Adolescent/

74 adolescen\#.mp.

7573 or 74

7666 and 72 and 75

7760 and 76

\section{Appendix 3. CINAHL search strategy}

1 exp Prospective Studies/

2 exp Case Control Studies/

3 exp Correlational Studies/

4 exp Nonconcurrent Prospective Studies/

5 exp Cross Sectional Studies/

6 (cohort adj (study or studies)).mp. [mp=title, subject heading word, abstract, instrumentation]

7 (observational adj (study or studies)).mp. [mp=title, subject heading word, abstract, instrumentation]

8 Randomized Controlled Trials.mp.

9 clinical trial.pt.

10 exp Clinical Trials/

11 (clin\$ adj25 trial\$).tw.

Exercises for adolescent idiopathic scoliosis (Review)

Copyright $\odot 2012$ The Cochrane Collaboration. Published by John Wiley \& Sons, Ltd. 
12 ((singl\$ or doubl\$ or trebl\$ or tripl\$) adj25 (blind\$ or mask\$)).tw.

13 exp PLACEBOS/

14 placebo\$.tw.

15 random\$.tw.

16 exp Study Design/

17 (latin adj square).tw.

18 exp Comparative Studies/

19 exp Evaluation Research/

20 Follow-Up Studies.mp.

21 exp Prospective Studies/

22 (control\$ or prospectiv\$ or volunteer\$).tw.

23 Animals/

24 or/ $1-22$

2524 not 23

26 Randomized Controlled Trials.mp.

27 clinical trial.pt.

28 exp Clinical Trials/

29 (clin\$ adj25 trial\$).tw.

30 ( (singl\$ or doubl\$ or trebl\$ or tripl\$) adj25 (blind\$ or mask\$)).tw.

31 exp PLACEBOS/

32 placebo\$.tw.

33 random $\$ . t w$.

34 exp Study Design/

35 (latin adj square).tw.

36 exp Comparative Studies/

37 exp Evaluation Research/

38 Follow-Up Studies.mp.

39 exp Prospective Studies/

40 (control\$ or prospectiv\$ or volunteer\$).tw.

41 Animals/

42 or $/ 26-40$

4342 not 41

44 exp SPINE/

45 exp Spinal Diseases/

$46 \exp$ SCOLIOSIS/

47 scoliosis.mp.

48 or/ $44-47$

49 exp Orthoses/

50 brace $\$$.mp.

51 bracing.mp.

52 or/49-51

53 exp Adolescence/

54 adolescen $\$ . \mathrm{mp}$.

5553 or 54

5648 and 52 and 55

5743 and 56

Exercises for adolescent idiopathic scoliosis (Review) 


\section{Appendix 4. Other database search strategies}

\section{CENTRAL}

\#1 MeSH descriptor Spinal Diseases explode all trees

\#2 MeSH descriptor Scoliosis explode all trees

\#3 scoliosis

\#4 (\#1 OR \#2 OR \#3)

\#5 MeSH descriptor Exercise explode all trees

\#6 exercis*

\#7 MeSH descriptor Exercise Therapy explode all trees

\#8 MeSH descriptor Exercise Movement Techniques explode all trees

\#9 MeSH descriptor Physical Therapy Modalities explode all trees

\#10 physiotherap*

\#11 MeSH descriptor Rehabilitation explode all trees

\#12 (\#6 OR \#7 OR \#8 OR \#9 OR \#10 OR \#11)

\#13 (\#4 AND \#12)

\section{SportDiscus}

S7 S6 and 1

S6 S5 or S4 or S3 or S2

S5 DE "REHABILITATION" (Explode thesaurus term)

S4 DE "PHYSICAL therapy" (Explode thesaurus term)

S3 DE "EXERCISE” (Explode thesaurus term)

S2 exercise

S1 scoliosis

\section{PEDro}

Simple search in titles and abstracts for "scolioisis"

\section{PsycInfo}

$(\mathrm{KW}=$ scoliosis $)$ and ((DE=("exercise" or "health behavior" or "movement therapy" or "physical fitness")) or $(\mathrm{KW}=\mathrm{exercise})$ or $(\mathrm{DE}=$ "physical therapy") or(DE="rehabilitation"))

\section{Index to Chiropractic Literature}

S1 Subject: "Scoliosis" OR All Fields:scoliosis

S2 Subject:"Exercise” OR Subject:"Exercise Therapy" OR All Fields:exercis*

S3 Subject: "Rehabilitation" OR All Fields:physiotherap*

S4 S2 OR S3

S5 S1 AND 54

\section{Appendix 5. Criteria for risk of bias assessment for RCTs and CCTs}

\section{Random sequence generation (selection bias)}

\section{Selection bias (biased allocation to interventions) due to inadequate generation of a randomised sequence}

There is a low risk of selection bias if the investigators describe a random component in the sequence generation process such as: referring to a random number table, using a computer random number generator, coin tossing, shuffling cards or envelopes, throwing dice, drawing of lots, minimisation (minimisation may be implemented without a random element, and this is considered to be equivalent to being random).

There is a high risk of selection bias if the investigators describe a non-random component in the sequence generation process, such as: sequence generated by odd or even date of birth, date (or day) of admission, hospital or clinic record number; or allocation by judgement of the clinician, preference of the participant, results of a laboratory test or a series of tests, or availability of the intervention. 


\section{Allocation concealment (selection bias)}

\section{Selection bias (biased allocation to interventions) due to inadequate concealment of allocations prior to assignment}

There is a low risk of selection bias if the participants and investigators enrolling participants could not foresee assignment because one of the following, or an equivalent method, was used to conceal allocation: central allocation (including telephone, web-based and pharmacy-controlled randomisation); sequentially numbered drug containers of identical appearance; or sequentially numbered, opaque, sealed envelopes.

There is a high risk of bias if participants or investigators enrolling participants could possibly foresee assignments and thus introduce selection bias, such as allocation based on: using an open random allocation schedule (e.g. a list of random numbers); assignment envelopes were used without appropriate safeguards (e.g. if envelopes were unsealed or non-opaque or not sequentially numbered); alternation or rotation; date of birth; case record number; or other explicitly unconcealed procedures.

\section{Blinding of participants}

\section{Performance bias due to knowledge of the allocated interventions by participants during the study}

There is a low risk of performance bias if blinding of participants was ensured and it was unlikely that the blinding could have been broken; or if there was no blinding or incomplete blinding, but the review authors judge that the outcome is not likely to be influenced by lack of blinding.

\section{Blinding of personnel/ care providers (performance bias)}

\section{Performance bias due to knowledge of the allocated interventions by personnel/care providers during the study}

There is a low risk of performance bias if blinding of personnel was ensured and it was unlikely that the blinding could have been broken; or if there was no blinding or incomplete blinding, but the review authors judge that the outcome is not likely to be influenced by lack of blinding.

Blinding of outcome assessor (detection bias)

\section{Detection bias due to knowledge of the allocated interventions by outcome assessors}

There is low risk of detection bias if the blinding of the outcome assessment was ensured and it was unlikely that the blinding could have been broken; or if there was no blinding or incomplete blinding, but the review authors judge that the outcome is not likely to be influenced by lack of blinding, or:

- for patient-reported outcomes in which the patient was the outcome assessor (e.g. pain, disability): there is a low risk of bias for outcome assessors if there is a low risk of bias for participant blinding (Boutron 2005)

- for outcome criteria that are clinical or therapeutic events that will be determined by the interaction between patients and care providers (e.g. co-interventions, length of hospitalisation, treatment failure), in which the care provider is the outcome assessor: there is a low risk of bias for outcome assessors if there is a low risk of bias for care providers (Boutron 2005)

- for outcome criteria that are assessed from data from medical forms: there is a low risk of bias if the treatment or adverse effects of the treatment could not be noticed in the extracted data (Boutron 2005)

Exercises for adolescent idiopathic scoliosis (Review) 


\section{Incomplete outcome data (attrition bias)}

\section{Attrition bias due to amount, nature or handling of incomplete outcome data}

There is a low risk of attrition bias if there were no missing outcome data; reasons for missing outcome data were unlikely to be related to the true outcome (for survival data, censoring unlikely to be introducing bias); missing outcome data were balanced in numbers, with similar reasons for missing data across groups; for dichotomous outcome data, the proportion of missing outcomes compared with the observed event risk was not enough to have a clinically relevant impact on the intervention effect estimate; for continuous outcome data, the plausible effect size (difference in means or standardised difference in means) among missing outcomes was not enough to have a clinically relevant impact on observed effect size, or missing data were imputed using appropriate methods (if dropouts are very large, imputation using even "acceptable" methods may still suggest a high risk of bias) (van Tulder 2003). The percentage of withdrawals and drop-outs should not exceed $20 \%$ for short-term follow-up and $30 \%$ for long-term follow-up and should not lead to substantial bias (these percentages are commonly used but arbitrary, not supported by literature) (van Tulder 2003).

\section{Selective Reporting (reporting bias)}

\section{Reporting bias due to selective outcome reporting}

There is low risk of reporting bias if the study protocol is available and all of the study's pre-specified (primary and secondary) outcomes that are of interest in the review have been reported in the pre-specified way, or if the study protocol is not available but it is clear that the published reports include all expected outcomes, including those that were pre-specified (convincing text of this nature may be uncommon).

There is a high risk of reporting bias if not all of the study's pre-specified primary outcomes have been reported; one or more primary outcomes is reported using measurements, analysis methods or subsets of the data (e.g. subscales) that were not pre-specified; one or more reported primary outcomes were not pre-specified (unless clear justification for their reporting is provided, such as an unexpected adverse effect); one or more outcomes of interest in the review are reported incompletely so that they cannot be entered in a metaanalysis; the study report fails to include results for a key outcome that would be expected to have been reported for such a study.

\section{Group similarity at baseline (selection bias)}

Bias due to dissimilarity at baseline for the most important prognostic indicators.

There is low risk of bias if groups are similar at baseline for demographic factors, value of main outcome measure(s), and important prognostic factors (examples in the field of back and neck pain are duration and severity of complaints, vocational status, percentage of patients with neurological symptoms) (van Tulder 2003).

\section{Co-interventions (performance bias)}

\section{Bias because co-interventions were different across groups}

There is low risk of bias if there were no co-interventions or they were similar between the index and control groups (van Tulder 2003).

\section{Compliance (performance bias)}

\section{Bias due to inappropriate compliance with interventions across groups}

There is low risk of bias if compliance with the interventions was acceptable, based on the reported intensity/dosage, duration, number and frequency for both the index and control intervention(s). For single-session interventions (e.g. surgery), this item is irrelevant (van Tulder 2003).

Exercises for adolescent idiopathic scoliosis (Review) 


\section{Intention-to-treat-analysis}

There is low risk of bias if all randomised patients were reported/analysed in the group to which they were allocated by randomisation.

\section{Timing of outcome assessments (detection bias)}

\section{Bias because important outcomes were not measured at the same time across groups}

There is low risk of bias if all important outcome assessments for all intervention groups were measured at the same time (van Tulder 2003).

\section{Other bias}

Bias due to problems not covered elsewhere in the table

There is a low risk of bias if the study appears to be free of other sources of bias not addressed elsewhere (e.g. study funding).

\section{Appendix 6. Criteria for risk of bias assessment for observational studies}

\section{Selection bias:}

1. Representativeness of the exposed cohort: Item is assessing the representativeness of exposed individuals in the community, not the representativeness of the sample of adolescents from a general population. Assess whether the sample is truly representative of the average adolescent with scoliosis; somewhat representative of the average adolescent with scoliosis; selected group of adolescents with scoliosis; no description of the derivation of the cohort. This item will be added in the 'Risk of bias' table as "other source of bias".

2. Selection of the non exposed cohort: Item is assessing the representativeness of non-exposed individuals in the same community as the exposed cohort that have been included in the study during the study period. Assess whether the sample has been drawn from the same community as the exposed cohort; drawn from a different source "no description of the derivation of the non-exposed cohort'. This item will be added in the 'Risk of bias' table as "other source of bias".

3. Ascertainment of exposure: Information in the study was obtained from a secure record (e.g. clinical records); structured interview; written self report; no description. This item will be added in the 'Risk of bias' table as "other source of bias".

4. Comparability of cohorts on the basis of the design or analysis: Either exposed and non-exposed individuals must be matched in the design and/or confounders must be adjusted for in the analysis. Statements of no differences between groups or that differences were not statistically significant are not sufficient for establishing comparability. If the relative risk for the exposure of interest is adjusted for the confounders listed, then the groups will be considered to be comparable on each variable used in the adjustment. Were most important prognostic factors matched? Yes/No. Were unmatched important prognostic factors adjusted for? Yes/No. This item will be assessed in the 'Risk of bias' table under the item "group similar at baseline“.

\section{Attrition bias:}

5. Complete follow-up: Assess if: all participants are accounted for; participants lost to follow-up unlikely to introduce bias (lost to follow-up < 5\%); participants lost to follow-up > 5\% and description provided of those lost. This item will be assessed in the 'Risk of bias' table under the item "incomplete outcome data".

Detection bias:

6. Independent blind assessment: Independent or blind assessment stated in the paper, or confirmation of the outcome by reference to secure records (x-rays, medical records, etc.), record linkage, or self-report; or no blinding; no description. This item will be assessed in the 'Risk of bias' table under the item "blinding of outcome assessor" 


\section{Appendix 7. Assessment of Clinial Relevance}

1. Are the patients described in detail so that you can decide whether they are comparable to those that you see in your practice?

2. Are the interventions and treatment settings described well enough so that you can provide the same for your patients?

3. Were all clinically relevant outcomes measured and reported?

4. Is the size of the effect clinically important?

5. Are the likely treatment benefits worth the potential harms?

\section{H I S T O R Y}

Protocol first published: Issue 2, 2009

Review first published: Issue 8, 2012

\section{CONTRIBUTIONS OF AUTHORS}

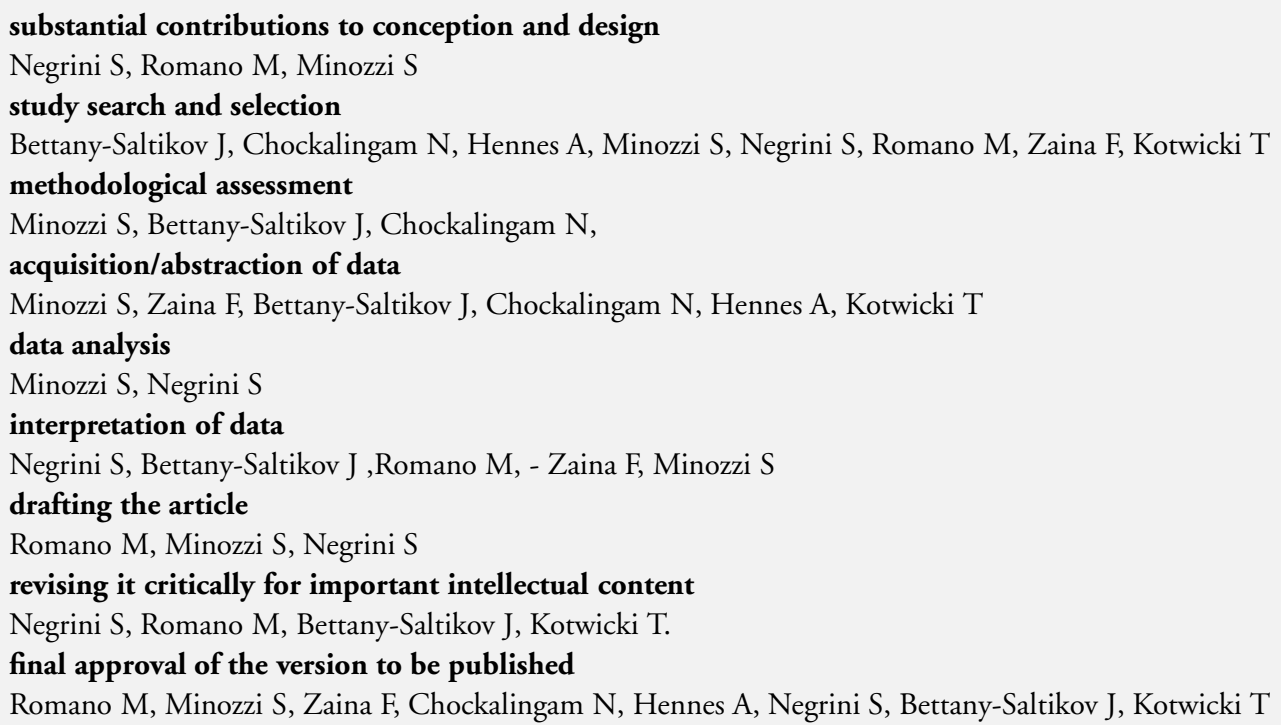

\section{DECLARATIONS OF INTEREST}

Many members of the review team have published widely in the field of scoliosis. One of the included studies (Negrini 2008a) was published by two authors of the review. Whenever a paper authored by a review author is considered, all decisions about the paper have been be made by the other review authors. 


\section{SOURCES OF SUPPORT}

\section{Internal sources}

- No sources of support supplied, Not specified.

\section{External sources}

- No sources of support supplied, Not specified. 